\title{
Role of AFB culture, histopathology and laparoscopy in female genital tuberculosis as a cause for chronic pelvic pain
}

\author{
Hemlata Sharma $^{1 *}$, Saurabh Sharma ${ }^{2}$, Neelam Bharadwaj ${ }^{1}$, Indira Lamba ${ }^{1}$
}

${ }^{1}$ Department of Obstetrics and Gynecology, SMS Medical College, Jaipur, Rajasthan, India
${ }^{2}$ Department of Pathology, Mahatma Gandhi Medical College and Hospital, Jaipur, Rajasthan, India

Received: 09 May 2017

Revised: 18 May 2017

Accepted: 03 June 2017

\section{*Correspondence:}

Dr. Hemlata Sharma,

E-mail: drhemlatasaurabh@gmail.com

Copyright: (c) the author(s), publisher and licensee Medip Academy. This is an open-access article distributed under the terms of the Creative Commons Attribution Non-Commercial License, which permits unrestricted non-commercial use, distribution, and reproduction in any medium, provided the original work is properly cited.

\section{ABSTRACT}

Background: As Female genital tuberculosis is an important cause of significant morbidity presenting mainly as infertility and chronic pelvic pain so the study was proposed to estimate the prevalence of genital tuberculosis in women with idiopathic chronic pelvic pain by AFB culture, histopathology and laparoscopy.

Methods: In this prospective study, we enrolled 65 women with idiopathic chronic pelvic pain. Diagnostic laparoscopy was done and Endometrial biopsy or menstrual blood was taken and sent for AFB culture and histopathological examination.

Results: In this study, the prevalence of genital TB in women with CPP was $35.38 \%$ (24/65) of which23 had gross findings of genital TB and one had pelvic congestion with positive result in AFB culture. Laparoscopic findings were normal in 13 out of 65 women $(20 \%)$ whereas these were abnormal in 52 women. Histological evidence of genital TB was reported in $12.5 \%$ (3/24) women. AFB culture reported positive in $16.66 \%$ (4/24) women.

Conclusions: Genital tuberculosis is an important cause for idiopathic chronic pelvic pain mainly in developing countries. AFB culture of menstrual blood is a simple, noninvasive test that is useful many times especially in unmarried girls or those who are not willing for invasive tests. Laparoscopy is an excellent tool in diagnosis of genital $\mathrm{TB}$ as it allows direct visualization.

Keywords: AFB culture, Chronic pelvic pain, Histopathology, Laparoscopy

\section{INTRODUCTION}

Chronic pelvic pain is a common gynecologic complaint that accounts for about $26 \%$ of outpatient visits to gynaecologist. ${ }^{1}$ CPP is responsible for about $40 \%$ of laparoscopies and 10-15\% of hysterectomies performed by gynaecologist. ${ }^{2}$ Global prevalence of CPP ranges from $4-43.4 \%$ with prevalence rates reported to be $15 \%$ and $43 \%$ in United states and India respectively. ${ }^{3}$ CPP is an intermittent or constant pain in lower abdomen or pelvis of at least 6months in duration not occurring exclusively with menstruation or intercourse and not associated with pregnancy. It is a symptom not a diagnosis. ${ }^{4}$ Because of shared interventions and functional interconnections between pelvic viscera, somatic structures and CNS, it has a complex pathophysiology and multifactorial etiology. Clinical examination alone is not conclusive in many patients, therefore imaging of pelvic organs needed. Laparoscopy is an excellent tool in evaluation of CPP. It enables not only confirmation of clinical and USG diagnosis, but also detects causes of pain in many, where these two are inconclusive.

Pelvic inflammatory disease is an important cause of CPP, of which genital tuberculosis (GTB) may present as $\mathrm{CPP}$ in $42-80 \%$ of cases the later being higher in 
developing countries. ${ }^{5,6}$ GTB represents $15-20 \%$ of extrapulmonary tuberculosis. ${ }^{7}$ GTB is mostly secondary infection acquired by hematogenous spread from an extagenital source such as pulmonary or abdominal tuberculosis. ${ }^{8}$ Fallopian tubes are affected in almost all cases $(95-100 \%)$ followed by endometrium and cervix..$^{9,10}$ GTB occurs in the reproductive age group $(15-45 \mathrm{yr}) .{ }^{11}$

Diagnosis of female genital TB is difficult due to it's paucibacillary nature and available conventional methods such as erythrocyte sedimentation rate (ESR), mantoux test and chest X-Ray have low sensitivity and specificity. Isolation of acid-fast bacilli (AFB) on smear and culture and presence of granulomas on histopathology of endometrial biopsy have the highest specificity for diagnosis of genital TB, but these tests lack sensitivity as the sampled site may not represent the infected area or due to cyclic shedding of endometrium. ${ }^{9}$

Rapid diagnostic methods such as BACTEC 460 mycobacteria growth inhibitor tube (MGIT) and specific gene probes have recently gained popularity. ${ }^{12}$ Polymerase chain reaction(PCR) for detecting mycobacterial DNA (mpt 64 gene) has high sensitivity and specificity and may indicate disease positivity even if the no. of bacteria present is as low as 1-10 organisms $/ \mathrm{mL} .{ }^{11}$

\section{METHODS}

This prospective study was conducted in department of obstetrics and gynecology, SMS hospital and Medical college, Jaipur from June 2016 to January 2017. A total of 65 women included in this study, attending gynecology outpatient department with complaint of chronic pelvic pain and not relieving with usual treatment.

Women who found to have a non-gynecological cause for chronic pelvic pain i.e. gastrointestinal, urinary cause were excluded from this study and referred to respective specialist for needful. Women with significant findings in ultrasonography like fibroid uterus as a cause for chronic pelvic pain were excluded. Only those with normal transvaginal ultrasonography were included in this study.

After taking consent, detailed history taken from all cases and after thorough clinical examination, all women underwent through investigations-Complete blood count, liver and renal function test, blood sugar, serum electrolyte, urine complete, erythrocyte sedimentation rate, chest X-Ray and ultrasonography-whole abdomen and pelvis and for uterus and adnexa.

Diagnostic laparoscopy was performed under general anaesthesia after pre anaesthetic check-up. Endometrial biopsy (premenstrual)or menstrual blood-whichever feasible, was taken and sent for AFB culture in Normal saline. Endometrial biopsy also sent for histopathological examination in formalin.

\section{Statistical analysis}

Data were collected and analyzed. Data were presented as percentages. A $p$ value of $<0.05$ was considered statistically significant.

\section{RESULTS}

A total of 65 women were enrolled in this study.

Table 1: Distribution of cases according to age group.

\begin{tabular}{|lll|}
\hline Age (year) & No. of women & $\%$ \\
\hline$<20$ & 2 & 3.07 \\
\hline $20-25$ & 15 & 23.07 \\
\hline $26-30$ & 26 & 40 \\
\hline $31-35$ & 16 & 24.61 \\
\hline$>35$ & 6 & 9.21 \\
\hline
\end{tabular}

Table 1 shows distribution of cases according to age group and suggests that mostly women 26 out of 65 (40\%) were of $26-30 \mathrm{yr}$ age, followed by $31-35 \mathrm{yr}$ (24.61\%) and $20-25 \mathrm{Yr}(23.07 \%) .6 / 65(9.21 \%)$ women were of $>35 \mathrm{yr}$ age. Only 2 women out of 65 (3.07\%) were of $<20 \mathrm{yr}$ age.

Table 2: Distribution of cases according to parity.

\begin{tabular}{|lll|}
\hline Parity & No. of women & $\%$ \\
\hline P0 & 20 & 30.7 \\
\hline Abortion & 6 & 9.23 \\
\hline P1 & 12 & 18.46 \\
\hline P2 & 18 & 27.69 \\
\hline$\geq$ P3 & 9 & 13.84 \\
\hline
\end{tabular}

Table 2 shows distribution of cases according to their parity. Mostly women with complaint of chronic pelvic pain were parous i.e. P1 or more. 20/65 (30.7\%) women were nulliparous. $6 / 65(9.23 \%)$ women had history of one or more abortion but no vaginal delivery. $9 / 65$ (13.84\%) women were $\mathrm{P} 3$ or more.

Table 3: Distribution of cases according to socioeconomic scale.

\begin{tabular}{|lll|}
\hline SES & No. of women & $\%$ \\
\hline II and III & 34 & 52.30 \\
\hline IV and V & 31 & 47.69 \\
\hline
\end{tabular}

Table 3 shows distribution of cases according to Kuppuswamy's scale and mostly women belonged to middle and lower socioeconomic group.

Table 4 shows distribution of cases according to duration of pain. 24/65 (36.92\%) women had chronic pelvic pain for 6-12 months.

21 women had similar complaints for 12-18months. $12 / 65(18.46 \%)$ had similar complaints for $18-24$ months. 
8/65 (12.30\%)women presented with complaint of chronic pelvic pain for $>2$ years.

Table 4: Distribution of cases according to duration of pain.

\begin{tabular}{|lll|}
\hline Duration of pain & No. of women & $\%$ \\
\hline $6-12 \mathrm{~m}$ & 24 & 36.92 \\
\hline $12-18 \mathrm{~m}$ & 21 & 32.30 \\
\hline $18-24 \mathrm{~m}$ & 12 & 18.46 \\
\hline$>2 \mathrm{yr}$ & 8 & 12.30 \\
\hline
\end{tabular}

Table 5: Distribution of cases according to associated complaints along with pelvic pain.

\begin{tabular}{|lll|}
\hline Associated complaints & No. of women & $\%$ \\
\hline Menstrual irregularity & & \\
\hline Oligomenorrhea & 18 & 27.69 \\
\hline Menorrhagia & 2 & 3.07 \\
\hline Amenorrhea & 5 & 7.69 \\
\hline Vaginal discharge & 32 & 49.23 \\
\hline Backache & 28 & 43.07 \\
\hline Dyspareunia & 25 & 38.46 \\
\hline Dysmenorrhea & 22 & 33.8 \\
\hline Infertility & 22 & 33.8 \\
\hline
\end{tabular}

Table 5 shows distribution of cases according to associated complaints along with pelvic pain. Most common associated complaint was vaginal discharge in $32 / 65$ (49.23\%) women. 28/65 (43.07\%) women had associated backache. 25 out of 65 women $(38.46 \%)$ had associated menstrual abnormality, of which mostly had oligomenorrhea $18 / 65(27.69 \%)$, followed by amenorrhea in 5 women $(3.07 \%)$ and menorrhagia in 2 women $(3.07 \%)$. Similar no. of women had associated dyspareunia i.e. $25 / 65(38.46 \%)$ women. 22 women out of $65(33.8 \%)$ presented with associated infertility and similar no. of women had associated dysmenorrhea.

Table 6: Distribution of cases according to various laparoscopic findings.

\begin{tabular}{|lll|}
\hline Laparoscopic findings & No. of women & $\%$ \\
\hline Normal & 13 & 20 \\
\hline Chronic PID & 30 & 46.15 \\
\hline Adhesions & 12 & 18.46 \\
\hline Endometriosis & 4 & 6.15 \\
\hline Pelvic congestion & 3 & 4.61 \\
\hline Ovarian cyst & 3 & 4.61 \\
\hline
\end{tabular}

Table 6 shows distribution of cases according to various laparoscopic findings. Laparoscopic findings were normal in $13 / 65(20 \%)$ women. 30 women out of 65 $(46.15 \%)$ had laparoscopic findings suggestive of chronic PID, followed by adhesions in 12 women (18.46\%), endometriosis in 4 women $(6.15 \%)$, pelvic congestion in 3 women $(4.61 \%)$ and ovarian cyst in 3 women $(4.61 \%)$. Out of 30 women of chronic PID, 23 women had findings suggestive of genital tuberculosis i.e. tubercles, caseation, beaded tubes, tubo-ovarian masses, fimbrial agglutination, hydrosalpinx, encysted peritonitis and peritubal adhesions. AFB culture was positive in 3 out of 23 women with findings of genital TB on laparoscopy. Histopathology reported evidence of tuberculosis in 3 out of 23 women with findings of genital TB on laparoscopy. One woman with findings suggestive of pelvic congestion on laparoscopy reported evidence of tuberculosis in AFB culture.

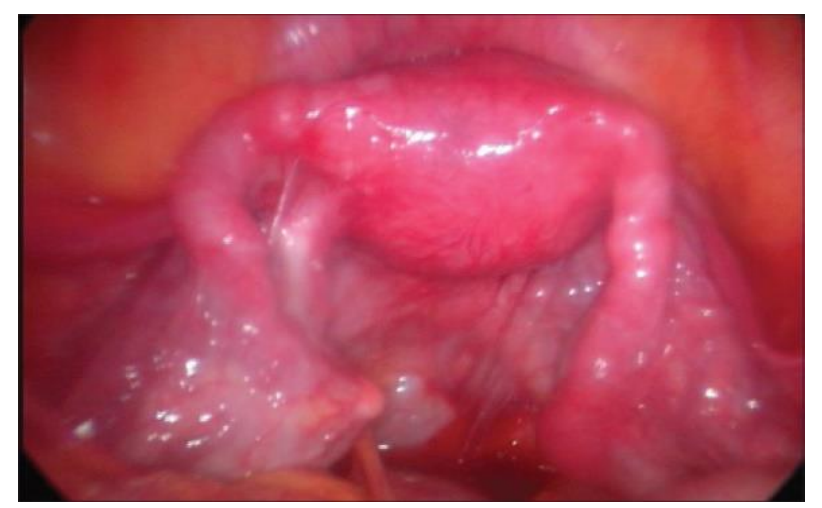

Figure 1: Bilateral beaded tubes studded with tubercles.

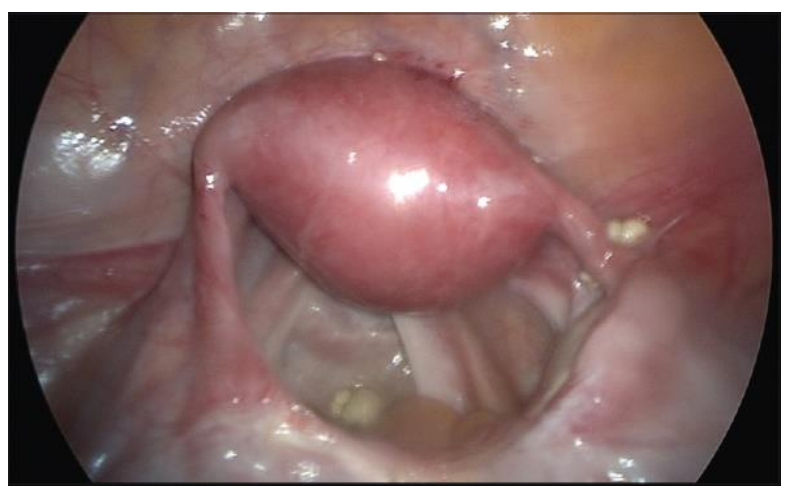

Figure 2: Caseous necrosis and bilateral tubo-ovarian mass.

The Figures 1, 2, and 3 shows various findings on laparoscopy that were suggestive of genital tuberuculosis. Figure 4 shows histopatholgical findings of tubercular endometritis.

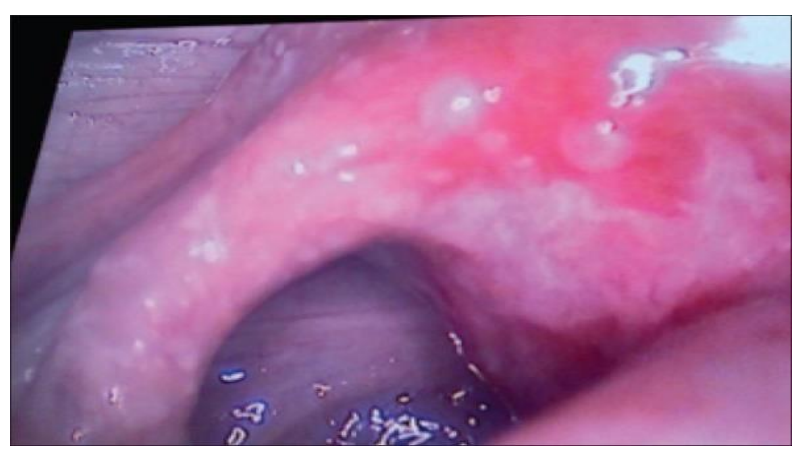

Figure 3: Fallopian tube studded with tubercles. 


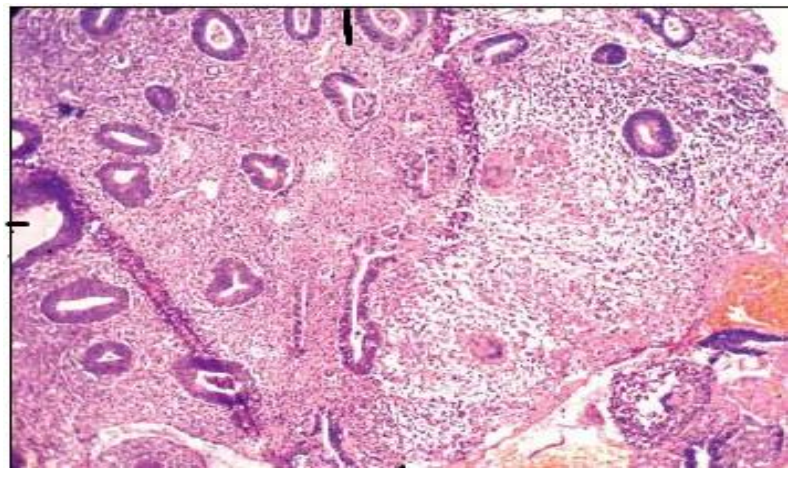

Figure 4: Epitheloid granulomas and giant cells of tubercular endometritis.

\section{DISCUSSION}

In present study, most cases belonged to 26-30yr age group and mostly were parous that is similar to other studies and mostly belonged to middle and lower

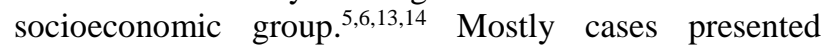
within 2 yrs of onset of pain. Menstrual disturbances were present in 25 women out of $65(38.46 \%)$ which is comparable to other studies. ${ }^{15}$ Oligomenorrhea was the commonest menstrual abnormality. Other associated complaints were vaginal discharge, dyspareunia and dysmenorrhea.

This study shows $35.38 \%$ prevalence of genital TB in women with CPP (24/65) of which 23 had gross findings of genital TB and one had pelvic congestion with positive result in AFB culture which is comparable with other studies in India. ${ }^{16}$ Some studies from India reported a lower prevalence rate of GTB 1.2-5\%.,17,18 By contrast one study from eastern Sudan reported a TB prevalence of $80 \% .^{19}$

Laparoscopic findings were normal in 13 out of 65 women $(20 \%)$ whereas these were abnormal in 52 women that are comparable with previous studies. ${ }^{20,21}$ Histological evidence of genital TB was reported in $12.5 \%(3 / 24)$ women that is comparable to other studies from India., ${ }^{9,22,23}$ AFB culture reported positive in $16.66 \%(4 / 24)$ women which is comparable to studies by Jindal and Bose. ${ }^{24,25}$ Rozati et al observed $7.8 \%$ and $11.5 \%$ detection rate of genital TB with AFB culture and histopathology respectively. ${ }^{26}$ Although these tests reported to show low sensitivity of only 30-35\% and culture requires upto 8 weeks to grow, bacteriological isolation of Mycobacteria is being considered the gold standard test. ${ }^{27-29}$

Negative results of endometrial biopsy do not rule out genital tuberculosis as sites other than endometrium like fallopian tube, pouch of douglas, ovaries may be involved without associated endometritis or the sampled site may not represent the infected area due to cyclic shedding of endometrium. One woman with findings suggestive of pelvic congestion on laparoscopy reported evidence of tuberculosis in AFB culture that may be an early sign of active genital tuberculosis or suggesting involvement of endometrium earlier than macroscopic visible changes in fallopian tubes.

\section{CONCLUSION}

Genital tuberculosis is an important cause for idiopathic chronic pelvic pain mainly in developing countries where it contributes to around one- third of cases. A single test is not sufficient to diagnose all the cases of genital TB. AFB culture of menstrual blood is an simple, non invasive test that is useful many times especially in unmarried girls or those who are not willing for invasive tests. Endometrial biopsy for histopathology is also an simple and cheaper test. These tests are highly specific though lack sensitivity. Laparoscopy is an excellent tool in diagnosis of genital TB as it allows direct visualization of pelvic viscera mainly fallopian tube that is the most common site for genital TB.

Funding: No funding sources Conflict of interest: None declared

Ethical approval: The study was approved by the Institutional Ethics Committee

\section{REFERENCES}

1. Ahangari A. Prevalence of chronic pelvic pain among women: an updated review. Pain physician 2014;17:E141-7.

2. ACOG Committee on practice, Bulletins Gynecology. ACOG Practice Bulletin No. 51. Chronic pelvic pain. Obstet Gynecol. 2004;103.

3. Latthe P, Latthe M, Say L, Gülmezoglu M, Khan KS. WHO systematic review of prevalence of chronic pelvic pain: a neglected reproductive health morbidity. BMC Public Health. 2006;6(1):177.

4. Royal College of Obstetricians and Gynecologists. The initial management of chronic pelvic pain. Green Top Guidelines 14. London. RCOG. 2012. Available at http://www.rcog.org.uk/files/rcog-corp/CPP-GTG. 2nd edition .pdf

5. Gatongi DK, Gitau G, Kay V, Ngwenya S, Lafong C, Hasan A. Female genital tuberculosis. Obstet Gynaecol. 2005;7(2):75-9.

6. Ali AA, Abdallah TM. Clinical presentation and epidemiology of female genital tuberculosis in eastern Sudan. Int J Gynecol Obstet. 2012;118(3):236-8.

7. Rajamaheshwari N. Pelvic tuberculosis. Int $\mathbf{J}$ Microbiol. 2008;27(4):361-3.

8. Gupta N, Sharma JB. Mittal S. Singh N, Mishra R. Kukreja. Genital tuberculosis in Indian infertility patients. Int J Gynecol Obstet. 2007;97(2):135-8.

9. Arora R, Sharma JB. Female genital TB-A diagnostic and therapeutic challenge. Indian $\mathbf{J}$ Tuberc. 2014;61:98-102 
10. Tripathy SN, Tripathy SN. Infertility and pregnancy outcome in female genital tuberculosis. Int J Gynecol Obstet. 2002;76(2):159-63.

11. Sharma JB. Tuberculosis and obstetric and gynecological practice. In Studd J, Tan SL, Chervenak FA, editors. $8^{\text {th }}$ ed. Progress in obstetrics and gynecology. Edinburgh, UK: Elsevier; 2008:395-427.

12. Neonakis IK, Gitti Z, Krambovitis E, Spandidos DA. Molecular diagnostic tools in mycobacteriology. J Microbiol Methods. 2008;75(1):1-1.

13. Goswami S, Chakraborty PS, Datta R. Laparoscopy in chronic pelvic pain. J Obstet Gynecol India. 2008;58(5):435-7.

14. Kamila G. Mukherji J, Gayan A. Different methods for evaluation of chronic pelvic pain. J Obstet Gynecol India. 2005;55:251-3.

15. Nagpal M, Pal D. Genital tuberculosis diagnostic dilemma in OPD patients. J Obstet Gynecol India. 2001;51:127-31.

16. Rajaram S, Gupta P, Gupta B, Kaur IR, Goel N. Laparoscopy in the diagnosis of tuberculosis in chronic pelvic pain. Int $\mathrm{J}$ Mycobacteriol. 5(2026)318-323.

17. Hebbar S, Chawla C. Role of laparoscopy in evaluation of chronic pelvic pain. J Minim Access Surg. 2005;1(3):116.

18. Sharma D, Dahiya K, Duhan N, Bansal R. Diagnostic laparoscopy in chronic pelvic pain. Arch Gynecol Obstet. 2011;283(2):295-7.

19. Iftikhar R. Outcome of laparoscopy in chronic pelvic pain. J Surg Pak. 2008;13:155-8.

20. Holloran-Schwartz MB. Surgical evaluation and treatment of the patient with chronic pelvic pain. Obstet Gynecol Clin North Am. 2014;41(3):357-69.

21. Kontoravdis A, Chryssikropoulos A, Hassiakos D, Liapis A, Zourlas PA. The diagnostic value of laparoscopy in 2365 patients with acute and chronic pelvic pain. Int J Obstet Gynecol. 1996;52:243-8.

22. Thangappah RB, Paramasivan $\mathrm{CN}$, Narayanan $\mathrm{S}$. Evaluating PCR, culture and histopathology in the diagnosis of female genital tuberculosis. Indian $\mathbf{J}$ Med Res. 2011;134(1):40.

23. Manjari M, Khanna S, Arora U, Kahlon SK, Gulati VL, Pushpa et al. Tuberculous endometritis in sterile females: a clinicopathological study. Indian J Tuberoc. 1995;42:227-8.

24. Jindal UN. An algorithmic approach to female genital tuberculosis causing infertility. Int $\mathrm{J}$ Tuberc Lung Dis. 2006;10(9):1045-50.

25. Bose M. Female genital tract tuberculosis:how long will it elude diagnosis? Indian J Med Res. 2011;134:13-4.

26. Rozati R, Sreenivsagar R, Rajeshwari CN. Evaluation of women with infertility and genital tuberculosis. J Obstet Gynecol India. 2006;56(5):423-6.

27. Shrivastava I, Bahatambare GS, Deshmukh AB, Rajpai P, Rajpai T Singh et al. Genital tuberculosis: evaluating microscopy, culture, histopathology and PCR for diagnosis all play their role. Int $\mathbf{J}$ Curr Microbiol Appl Sci. 2014;3(4):439-45.

28. Bhanu NV, Singh UB, Chakraborty M, Suresh N, Arora J, Rana $\mathrm{T}$ et al. Improved diagnostic value of PCR in diagnosis of female genital tuberculosis leading to infertility. J Med Microbiol. 2005;54(Pt 10):927-31.

29. Botha MH, Van der Merwe FH. Female genital tuberculosis: CPD. S Afr Fam Pract. 2008;50(5)12-6.

Cite this article as: Sharma $\mathrm{H}$, Sharma $\mathrm{S}$, Bharadwaj N, Lamba I. Role of AFB culture, histopathology and laparoscopy in female genital tuberculosis as a cause for chronic pelvic pain. Int J Reprod Contracept Obstet Gynecol 2017;6:3091-5. 